\title{
A Low-Power-Consumption Out-of-Plane Electrothermal Actuator
}

\author{
David Girbau, Marco Antonio Llamas, Jasmina Casals-Terré, Xavier Simó-Selvas, \\ Lluís Pradell, Member, IEEE, and Antonio Lázaro
}

\begin{abstract}
This paper proposes a new vertical electrothermal actuator. It can be considered as a hybrid between the traditional in-plane buckle-beam actuator and the vertical hot-cold actuator. It is here referred to as vertical buckle beam. At identical dimensional and bias conditions, it features a displacement larger than that of other vertical electrothermal actuators proposed so far in the literature. The actuator performance is demonstrated by means of an analytical model along with finite-element analysis. It is applied as a driving element in parallel-plate capacitors, where it is validated and its advantages in terms of power consumption are demonstrated empirically.

[2006-0115]
\end{abstract}

Index Terms-Buckle-beam actuator, out-of-plane (vertical) and in-plane electrothermal actuators.

\section{INTRODUCTION}

$\mathbf{O}$ UT-OF-PLANE and in-plane electrothermal actuators have demonstrated capabilities to be key elements in a number of applications such as micromirrors (for display and telecommunication), optical and RF switching, microlens scanners, microengines, microrelays, and variable capacitors [1]-[8]. High-displacement capability with low actuation voltage is their main characteristic. Concerning out-of-plane actuators, several topologies have been presented in the literature so far. The bimorph actuator [9], [10] is based on a bilayer structure, which is composed of two materials whose coefficients of thermal expansion differ from each other. If an electrical current is applied, it results in a temperature change, giving rise to a displacement, since the expansion of both layers is different. The vertical hot-cold structure is proposed in [11]; the hot arm is placed over the cold arm, resulting in a downward motion when a voltage is applied. A third vertical actuator is proposed in [7] and [12], which consists of a coplanar structure

Manuscript received June 21, 2006; revised January 3, 2007. This work was supported by the Ministerio de Ciencia y Tecnología (MCYT) under Spanish Government Project ESP2004-07067-C03-03. Subject Editor C. Ahn.

D. Girbau is with the Department of Signal Theory and Communications, Universitat Politécnica de Catalunya, 08034 Barcelona, Spain, and also with the Universitat Autónoma de Barcelona, 08193 Bellaterra, Spain (e-mail: DGirbauSala@tsc.upc.edu).

M. A. Llamas and L. Pradell are with the Department of Signal Theory and Communications, Universitat Politécnica de Catalunya, 08034 Barcelona, Spain (e-mail:marco@tsc.upc.edu; pradell@tsc.upc.edu).

J. Casals-Terré and X. Simó-Selvas are with the Department of Mechanical Engineering, Universitat Politécnica de Catalunya, 08226 Terrassa, Spain (e-mail: Jasmina.casals@upc.edu; xavi.simo@gmail.com).

A. Lázaro is with the Department of Electronics, Electrical and Automatics Engineering, University Rovira i Virgili, 43007 Tarragona, Spain (e-mail: antonioramon.lazaro@urv.net).

Color versions of one or more of the figures in this paper are available online at http://ieeexplore.ieee.org.

Digital Object Identifier 10.1109/JMEMS.2007.896714 made up of three beams with two possible topologies: a hot arm in the middle and two cold arms at both sides, or a cold arm in the middle and two hot arms at both sides. The arms are connected at one end and individually anchored to substrate at the other end. In this way, the arms' different expansion results into an out-of-plane displacement. Another vertical electrothermal actuator is proposed in [13]. It consists of four parallel identical beams connected at their free ends and anchored at the others. The inner and the outer beams are located at different planes by two steps placed in the free ends of the inner ones. When a current flows through the inner beams, they experience a thermal expansion larger than that at the outer beams and, since the beams are not coplanar, this gives rise to a torque that induces an upward motion. On the contrary, when a current flows through the outer beams, the actuator experiences a downward motion. Finally, another bidirectional vertical electrothermal actuator is proposed in [14]. In this case, the actuator has two superposed U-shaped beams anchored at the substrate separately at one end and connected at the other end through a via. It can move upward and downward, depending on where the voltage is applied, the top $\mathrm{U}$ or the bottom $\mathrm{U}$.

This paper proposes a new low-power-consumption out-ofplane electrothermal actuator, which is referred to here as vertical buckle beam (VBB) [see Fig. 1(a)]. The VBB actuator can be qualitatively considered as a hybrid between the traditional in-plane buckle-beam (V-shaped) actuator [15], [16] and the vertical hot-cold actuator [11]. Its main advantage with respect to other proposed electrothermal out-of-plane actuators [13], [14] is the increased displacement for the same dimensions and dc power consumption conditions since it provides a higher in-plane force due to the prebending angle $\varphi$, resulting in higher out-of-plane forces. Furthermore, in contrast to [11], the $\mathrm{dc}$ power consumption is optimized (i.e., the efficiency increases) since no current goes through the cold arm (as in [13] and [14]).

The VBB proposed is manufactured as the driving element of a parallel-plate RF capacitor and validated empirically through the measurement of the capacitor tuning range for several actuator prebending angles $\varphi$. The reason a parallel-plate capacitor is chosen as a demonstrator is because of its inherent tuning range limitation when it is driven by electrostatic actuators [17], [18]. Indeed, a number of design techniques to enlarge its tuning range have been proposed so far [17], [19], and the use of electrothermal actuators as driving elements [7], [8], [12], [14], [18] has proven to be a successful solution. Moreover, electrothermal actuators are good candidates to improve $\mathrm{RF}$ power handling and intermodulation distortion in $\mathrm{RF}$ 


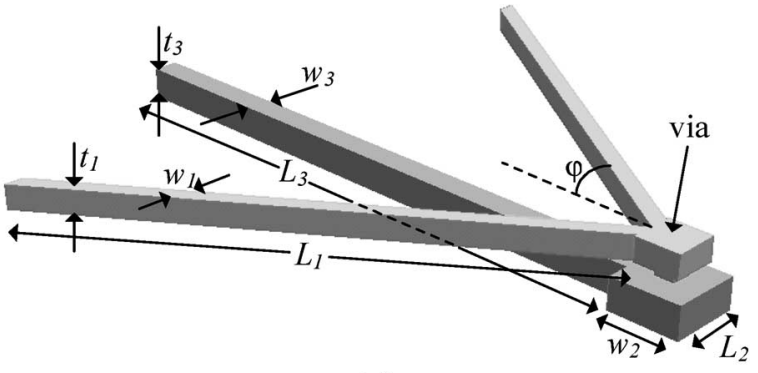

(a)

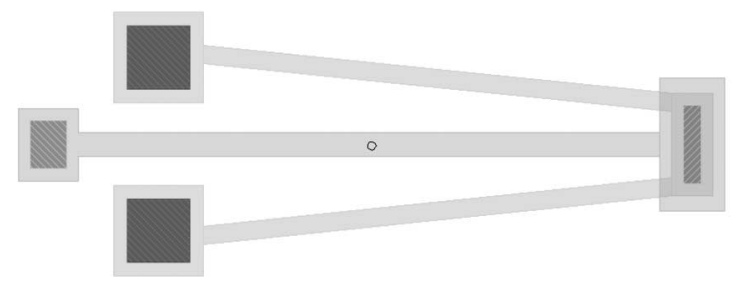

(b)

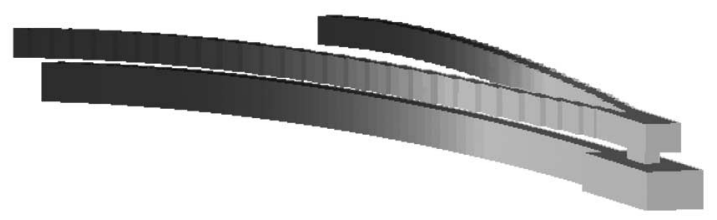

(c)

Fig. 1. (a) Three-dimensional view of the VBB with its main dimensions. (b) VBB 2-D mask $\left(\varphi=5^{\circ}\right)$. (c) VBB at an actuated position.

microelectromechanical system (MEMS) capacitors [20], [21], since they usually have a large vertical stiffness constant. In [8], a tunable capacitor based on an electrothermal actuator is proposed. The actuator is a lateral hot-cold type, which, with a suitable design, transforms its motion into an upward and downward displacement of a mobile membrane, which is, in turn, the capacitor top electrode. A capacity variation of 4.25:1 $(0.8-3.4 \mathrm{pF})$ was measured with an actuation voltage smaller than $3 \mathrm{~V}$. The vertical thermal actuator proposed in [7] and [12] is applied to the control of a parallel-plate capacitor, achieving a tuning range of 2.7:1. The bidirectional vertical electrothermal actuator presented in [14] was applied to a parallel-plate capacitor, tuning its capacity from 5.04 to $2.944 \mathrm{pF}$ (1.71:1), with an actuation voltage of $5 \mathrm{~V}$. Reference [18] proposes four U-shaped actuators as driving elements in a parallel-plate capacitor, with a variation of 2.21:1 (2.9-6.4 pF).

This paper is organized as follows. The VBB actuator operation is described in Section II; an analysis follows in Section III, describing a theoretical model for the actuator operation, which is validated through finite-element analysis (FEA) simulations. In Section IV, the proposed VBB actuator is applied as a driving element in a parallel-plate RF capacitor, experimentally tested, and validated.

\section{VBB ACTUATOR}

Fig. 1(a) shows a 3-D view of the VBB actuator with its main dimensions. It is implemented with the commercial surface micromachining fabrication process PolyMumps [22]. The VBB is made up of a V-shaped actuator in the top polysilicon layer (Poly2: see [22]), with a constrained motion in plane due to a straight beam in the bottom polysilicon layer (Poly1: see [22]). The V-shaped actuator is anchored to the substrate at both ends and connected through a Poly1-Poly2-via to the end of the straight beam, which is, in turn, connected to the substrate at the other end, as shown in Fig. 1(b). When a voltage is applied to the V-shaped beam, the current flows through the top layer only, which expands in-plane due to an increase in its temperature. The bottom beam, which is not affected by the applied voltage (cold arm), causes the actuator tip to deflect downward to the substrate, as shown in Fig. 1(c). The prebending angle $\varphi$ controls the actuator displacement.

The U-shaped actuator proposed in [14] may qualitatively be considered as a particular case of the VBB, when the two buckle-beam arms become parallel. The main difference between VBB and U-shaped actuators is the lack of the bottom $\mathrm{U}$ in the VBB (which is replaced with a cantilever beam, with a much smaller stiffness constant). The bottom U permits bidirectionality, but it decreases the displacement. The one proposed in this paper is unidirectional. However, when it is applied to RF capacitors- the case for the actuator proposed here- unidirectional displacement is enough, as the U-shaped actuator bidirectional capability can be compensated by increasing the initial gap and actuator displacement, increasing $\varphi$.

\section{VBB Analytical Thermomechanical Model}

To model the behavior of the VBB actuator, the physical analysis of the system has been divided into two parts: the thermal model and the mechanical model. This is shown in the block diagram of Fig. 2. The inputs to the models are the geometry of the VBB actuator and the voltage $V$ applied. This voltage causes an average distribution of temperature along the V-shaped beam $(\Delta T)$. This thermal load is used to calculate the in-plane deflection of the V-shaped beam $(\Delta L)$ [23], which, due to restrictions imposed by beam 3, produces the out-ofplane deflection $(\delta)$. Using this model, there are two basic optimization strategies to increase displacement: either, for given dimensions of the actuator, increase the prebending angle $\varphi$ or change the dimensions of the V-shaped beam (basically, increase $L_{1}$ ) until the desired deflection is obtained. The thermal and mechanical models are presented in the next sections.

\section{A. Thermal Analysis}

The actuator motion is achieved through material thermal expansion. Since the VBB has been fabricated with PolyMumps and considering the hypothesis verified in [14] and [23] for sacrificially released devices, most of the heat is dissipated toward the substrate through anchors. The VBB electrothermal analysis can be simplified as a 1-D heat transfer problem. It is assumed that the current flows through the V-shaped beam, which is modeled as three microbeams connected in series. The coordinate system used in the thermal analysis is shown in Fig. 3. $L_{1}, L_{2}$, and $L_{3}$ are, respectively, the lengths of the V-shaped half beam, the junction beam, and the bottom (cold) arm.

As stated in [14], under steady-state conditions and considering only heat conduction, the heat flow in one differential 


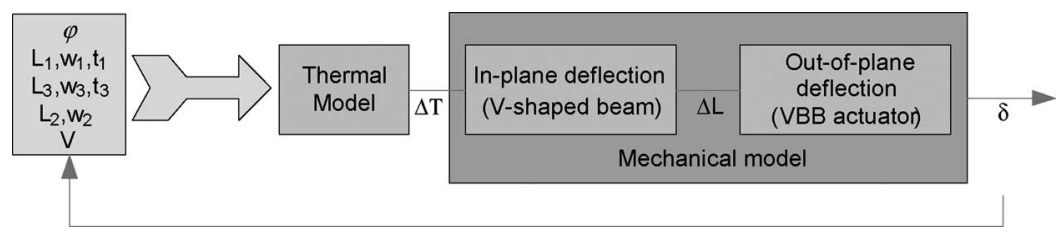

Fig. 2. VBB actuator design flow using the thermomechanical model presented in Section III.

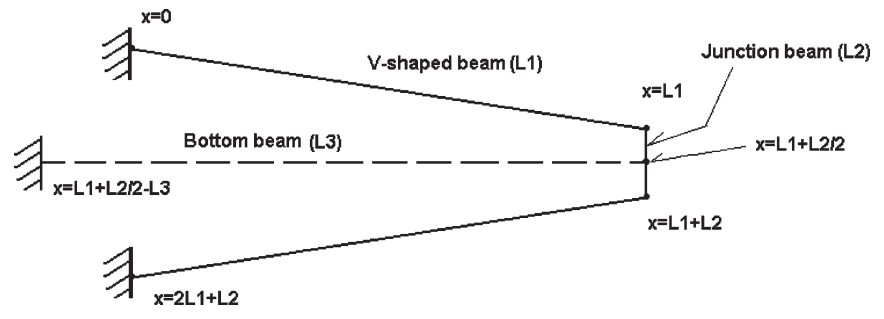

Fig. 3. One-dimensional coordinate system.

element $(d x)$ of the $\mathrm{V}$-shaped beam is ruled by the following equation:

$$
-\left.k \frac{\partial T}{\partial x}\right|_{x}+\dot{q}_{j} \cdot d x=-\left.k \frac{\partial T}{\partial x}\right|_{x+d x}
$$

where $\dot{q}_{j}$ is the ohmic heat produced in a cubic unit of beam material, and $k$ is the thermal conductivity. Equation (1) can be simplified by applying Taylor series expansion as follows:

$$
k \frac{\partial^{2} T}{\partial x^{2}}+\dot{q}_{j} \cdot d x=0 .
$$

The general solution to the temperature distribution for the $j$ th element of the V-shaped beam becomes

$$
\begin{aligned}
& T_{1}(x)=-\frac{\dot{q}_{1}}{2 k} x^{2}+C_{11} x+C_{12} \\
& T_{2}(x)=-\frac{\dot{q}_{2}}{2 k} x^{2}+C_{21} x+C_{22} \\
& T_{3}(x)=C_{31} x+C_{32}
\end{aligned}
$$

where $T_{1}(x), T_{2}(x)$, and $T_{3}(x)$ are temperature distributions in the $\mathrm{V}$-shaped beams, the junction beam, and the cold beam, respectively. Since no current goes to the bottom beam, the temperature distribution along it is a simple linear heat conduction problem. Coefficients $C_{11}, C_{12}, C_{21}, C_{22}, C_{31}$, and $C_{32}$ are integration constants that are solved by considering the following set of boundary conditions (6)-(11):

$$
T_{1}(0)=T_{s}
$$

which forces temperature in $x=0$ to be the substrate temperature $T_{s}$. The temperature at the beginning of the junction beam has to be the same as the $\mathrm{V}$-shaped end temperature, i.e.,

$$
T_{2}\left(L_{1}\right)=T_{1}\left(L_{1}\right)
$$

The heat flux conservation at the junction of each beam yields

$$
\left.\frac{d T_{1}}{d x}\right|_{x=L_{1}} A_{1}=\left.\frac{d T_{2}}{d x}\right|_{x=L_{1}} A_{2}
$$

where $A_{j}$ and $L_{j}$ are the beam cross-sectional areas and lengths, respectively.

The temperatures at the center point of the mechanism for the junction beam and for the cold beam are the same, i.e.,

$$
T_{3}\left(L_{1}+\frac{L_{2}}{2}\right)=T_{2}\left(L_{1}+\frac{L_{2}}{2}\right) .
$$

The heat fluxes in the junction beam and the cold beam are the same, i.e.,

$$
\left.\frac{d T_{2}}{d x}\right|_{x=L_{1}+\frac{L_{2}}{2}} A_{2}=\left.\frac{d T_{3}}{d x}\right|_{x=L_{1}+\frac{L_{2}}{2}} \cdot \frac{A_{3}}{2} .
$$

Since the VBB thermal actuator is symmetric along its center line, the temperature distribution is also symmetrical, and the cold beam has to be considered with a half cross section, because it is shared between the two symmetric parts.

It is assumed that the cold beam anchor temperature is the same as the substrate temperature $\left(T_{s}\right)$, i.e.,

$$
T_{3}\left(L_{1}+\frac{L_{2}}{2}-L_{3}\right)=T_{s}
$$

The ohmic heat power generated in a volumetric unit for each beam is

$$
\dot{q}_{1}=\frac{i^{2}}{A_{1}^{2} \sigma} \quad \dot{q}_{2}=\frac{i^{2}}{A_{2}^{2} \sigma}
$$

where $A_{1}$ and $A_{2}$ are cross-sectional areas, $\sigma$ is the electric conductivity, and $i$ is the current through the beam, which is given by

$$
i=\frac{V \sigma}{\left(\frac{2 L_{1}}{A_{1}}+\frac{L_{2}}{A_{2}}\right)}
$$

where $V$ is the applied voltage between anchors, and $L_{j}$ are the beam lengths. Using the boundary conditions to solve (3)-(5), the temperature distribution of a VBB actuator with dimensions in Table $\mathrm{I}$ is plotted in Fig. 4. The maximum temperature appears near the end of the V-shaped beam rather than at the end because the bottom beam acts as a heat sink. The dimensions in Table I have been chosen to optimize the power consumption and actuator area for the required displacement in the parallelplate capacitor presented in Section IV.

\section{B. Mechanical Analysis}

Since both beams (top and bottom) are subjected to compression/traction due to the thermal load, which causes lateral bending moment, it results in an out-of-plane motion. The VBB 
TABLE I

Simulated ACTUATOR Dimensions

\begin{tabular}{llc}
\hline \hline Dimension & & Value \\
& & \\
\hline \multirow{2}{*}{ Buckle-beam } & Arm length $\left(L_{1}\right)$ & $165 \mu \mathrm{m}$ \\
& Arm width $\left(w_{1}\right)$ & $6 \mu \mathrm{m}$ \\
& Arm thickness $\left(t_{1}\right)$ & $1.5 \mu \mathrm{m}$ \\
\hline \multirow{2}{*}{ Bottom } & Length $\left(L_{3}\right)$ & $195 \mu \mathrm{m}$ \\
cantilever beam & Width $\left(w_{3}\right)$ & $8 \mu \mathrm{m}$ \\
& Thickness $\left(t_{3}\right)$ & $2 \mu \mathrm{m}$ \\
\hline \multirow{2}{*}{ Junction } & Length $\left(L_{2}\right)$ & $14 \mu \mathrm{m}$ \\
& Width $\left(w_{2}\right)$ & $11 \mu \mathrm{m}$ \\
\hline \hline
\end{tabular}

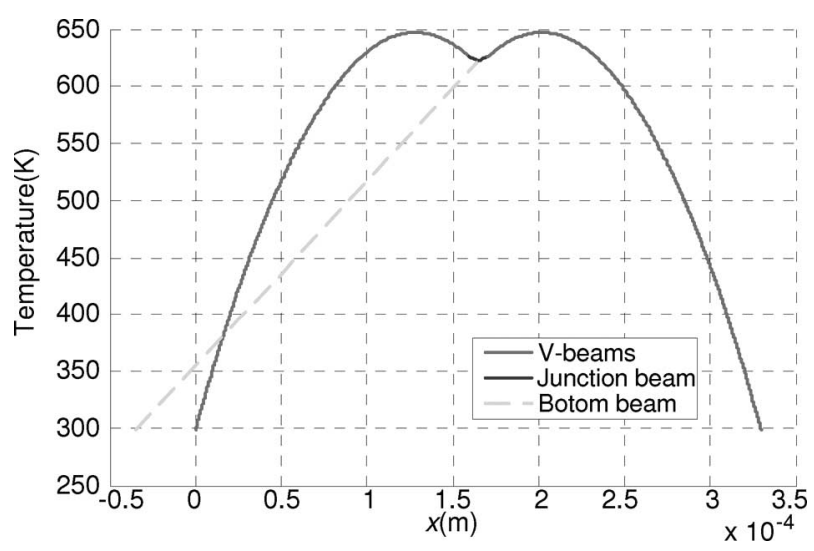

Fig. 4. Temperature distribution for $2.5 \mathrm{~V}$ applied.
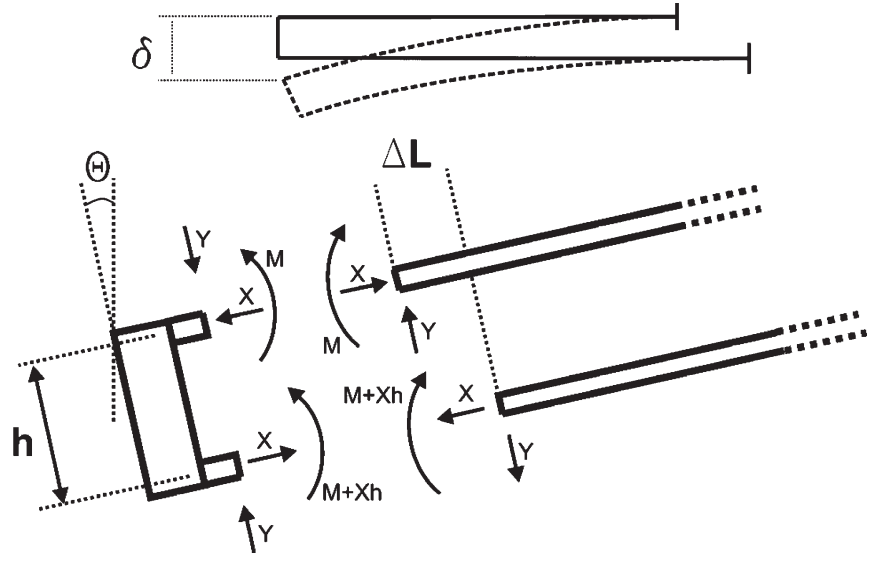

Fig. 5. Schematic view of the reaction forces and torques at the junction point.

actuator is modeled as a 1-D Euler-beam model. Fig. 5 shows a cross-section of the VBB actuator at its symmetry plane with reaction forces and moments. The input to the mechanical model is the average temperature of the actuator arms, which is derived from the previous analysis.

The out-of-plane displacement $\delta$ is due to an in-plane differential displacement between the top V-shaped beam and the bottom cold beam. Equation (14) defines the in-plane difference of thermal expansion that causes the out-of-plane motion $\delta$ as follows:

$$
\Delta L=\Delta L_{v}-\Delta L_{3}
$$

where $\Delta L_{v}$ is the in-plane displacement of the $\mathrm{V}$-shaped beam, which is analyzed by Enikov et al. [23]. The net enlargement of

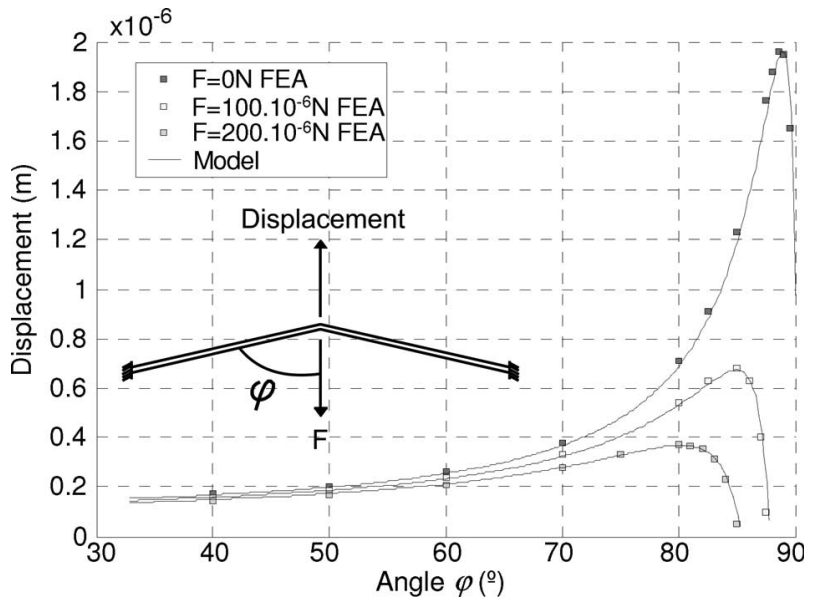

Fig. 6. In-plane free displacement analytical model [23] compared to FEA simulation results for the manufactured V-shaped beam. An average temperature increase of $300^{\circ} \mathrm{C}$ is considered.

the bottom beam is given by thermal expansion and compression of the beam as

$$
\Delta L_{3}(X)=\alpha \cdot L_{3} \cdot \Delta \bar{T}_{3}+\frac{X L_{3}}{E A_{3}}
$$

where $\alpha$ is the thermal expansion coefficient, $X$ is the axial load (see Fig. 5), $E$ is the Young modulus, and $\Delta \bar{T}_{3}$ is the average temperature increase of the bottom beam (16), which is defined by

$$
\Delta \bar{T}_{3}=\frac{\int_{0}^{L_{3}}\left(T_{3}(x)-T_{s}\right) d x}{L_{3}} .
$$

Fig. 6 shows the in-plane free displacement of a V-shaped actuator. It can be seen that as $\varphi$ increases, the in-plane motion is also increased, up to $\varphi$ angles where the V-shaped actuator is so open that, instead of acting as two bended beams, the beam acts as one slender beam and starts buckling due to the thermal load. In a VBB actuator, a compression force $F$ acts against displacement, limiting the tip in-plane motion and lowering the buckling point. However, even for high in-plane forces, the displacements for large $\varphi$ are higher than for small $\varphi$, if $\varphi$ angle is kept below $80^{\circ}$.

Generally, the V-shaped thermal actuator has a nonlinear behavior, but in the displacements range where the VBB works (less than $1 \mu \mathrm{m}$ ) and for the tested angles, the displacement can be approximated, with less than $0.1 \%$ error, by a linear behavior defined by

$$
\Delta L_{v}(X)=\Delta L_{v}(0)-r_{v} \cdot X
$$

where $\Delta L_{v}(0)$ is the displacement of a $\mathrm{V}$-shaped beam without an in-plane external load, and $r_{v}$ is the linearization of the displacement of the $\mathrm{V}$-shaped beam due to the compression force. These two values are obtained for each average temperature using Enikov's model [23].

Applying (15) and (17) to (14), $\Delta L$ becomes

$\Delta L(X)=\Delta L_{v}(0)-r_{v} \cdot X-\alpha \cdot L_{3} \cdot \Delta \bar{T}_{3}-\frac{X L_{3}}{E A_{3}}$. 
If the junction is considered incompressible due to its short length compared to the length of the bending beams, both the $\mathrm{V}$-shaped and bottom beams are constrained to have the same vertical deflection $\delta$, i.e.,

$$
\delta=\delta_{1}=\delta_{3}
$$

and the same rotation angle $\theta$, which is approximated to its tangent as

$$
\theta_{1}=\theta_{3}=\theta \approx \frac{\Delta L}{h}
$$

where $h$ is the out-of-plane distance between the bottom and top layers. Applying the Euler-beam theory, the VBB out-of-plane displacement is

$$
\begin{aligned}
& \delta=\delta_{1}=\frac{Y L_{1}^{3}}{3 E\left(2 I_{1}\right)}+\frac{\cos \varphi \cdot M \cdot L_{1}^{2}}{2 E\left(2 I_{1}\right)} \\
& \delta=\delta_{3}=-\frac{Y L_{3}^{3}}{3 E I_{3}}-\frac{(M+X \cdot h) \cdot L_{3}^{2}}{2 E I_{3}}
\end{aligned}
$$

where $Y$ and $M$ are the vertical force and the bending moment, respectively (see Fig. 5). The angle rotated by the end point of the two beams is

$$
\begin{gathered}
-\theta=-\theta_{1}=\frac{Y L_{1}^{2}}{2 E\left(2 I_{1}\right)}+\frac{\cos \varphi \cdot M \cdot L_{1}}{E\left(2 I_{1}\right)} \\
\theta=\theta_{3}=\frac{Y L_{3}^{2}}{2 E I_{3}}+\frac{(M+X \cdot h) L_{3}}{E I_{3}} .
\end{gathered}
$$

Note that, in the V-shaped beam equations, $I_{1}$ is the inertia moment of the V-shaped beams, the bending moment is projected in the out-of-plane direction $(\cos \varphi)$, and $I_{3}$ is the inertia moment of the bottom beam (cold arm).

From (18), (20)-(22), for a given average temperature, the vertical free displacement and rotation angle due to the thermal load can be calculated. The displacement $\delta$ is

$$
\delta=\frac{N_{1} \cos \psi}{N_{2} \cos \psi+N_{3}}\left(\Delta L_{v}(0)-\Delta L_{3}(0)\right) .
$$

The rotation angle $\theta$ is

$$
\theta=\frac{P_{1} \cos \psi}{P_{2} \cos \psi+P_{3}}\left(\Delta L_{v}(0)-\Delta L_{3}(0)\right) .
$$

$N 1, N 2, N 3, P_{1}, P_{2}$, and $P_{3}$ are given in the Appendix.

The above analytical model has been verified using FEA simulations. To compare the FEA results (maximum displacement) to the analytical model results, the junction solid rigid rotation has to be added to the displacement calculated from (23) as follows:

$$
\delta_{\mathrm{Max}}=\delta+\sin (\theta) w_{2}
$$

where $w_{2}$ is the junction width.

Plotting (25) for different prebending angles, it can be appreciated that the vertical displacement is increased as the prebending angle $\varphi$ increases. In Fig. 7, the analytical vertical displacement obtained from (25) is compared to the FEA

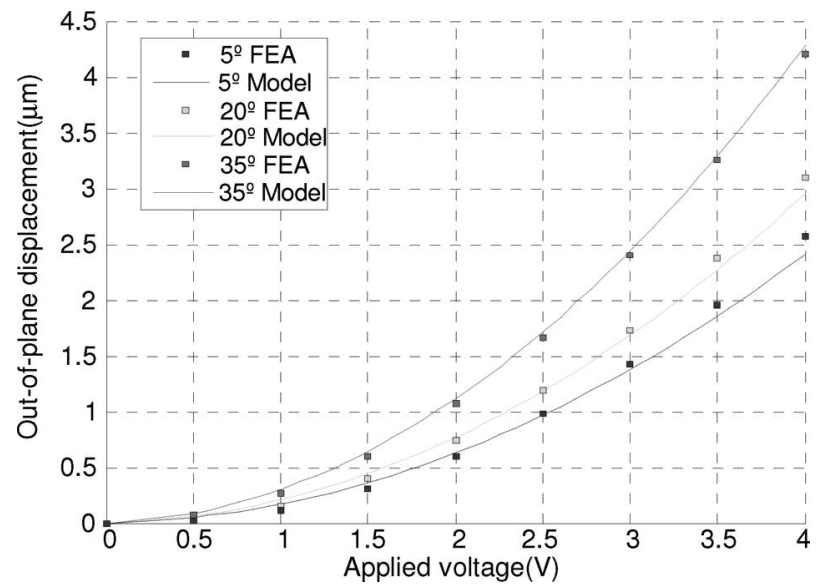

Fig. 7. Comparison between the analytical model proposed and the FEA simulations done with CoventorWare.

TABLE II

POLYSILICON PROPERTIES

\begin{tabular}{ll}
\hline \hline Property & Value @300 ${ }^{\circ} \mathrm{K}$ \\
\hline Young modulus $(E)$ & $1.65 \times 10^{5} \mathrm{MPa}$ \\
Residual stress & $12.086 \mathrm{MPa}$ \\
Thermal expansion coefficient $(\alpha)$ & $2.5 \times 10^{-6} 1 / \mathrm{K}$ \\
Thermal conductivity $(k)$ & $6.5 \times 10^{7} \mathrm{pW} / \mu \mathrm{mK}$ \\
Electrical conductivity $(\sigma)$ & $4.65 \times 10^{10} \mathrm{pS} / \mu \mathrm{m}$ \\
Specific heat & $7.2 \times 10^{14} \mathrm{pJ} / \mathrm{kgK}$ \\
\hline \hline
\end{tabular}

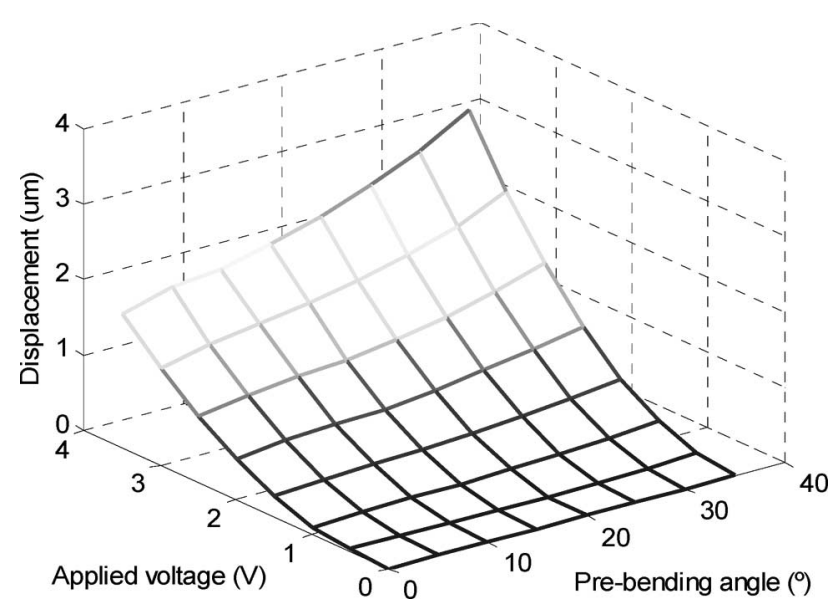

Fig. 8. Actuator free displacement as a function of the prebending angle $\varphi$ and the applied voltage.

(CoventorWare) simulations. Table II lists the values for the polysilicon properties assumed in the simulations.

Fig. 8 shows the VBB simulated free displacement as a function of the applied voltage $V$ and the prebending angle $\left(0^{\circ}<\varphi<35^{\circ}\right)$ using CoventorWare, whereas Fig. 9 shows the maximum temperature at the actuator.

If the actuator is coupled to a device, an external force will be applied, acting against the thermal deflection $\delta$. Considering small deflections of the VBB actuator, the total deflection becomes

$$
\delta_{T}=\delta+\frac{F_{\mathrm{ext}}}{k_{\mathrm{VBB}}}
$$




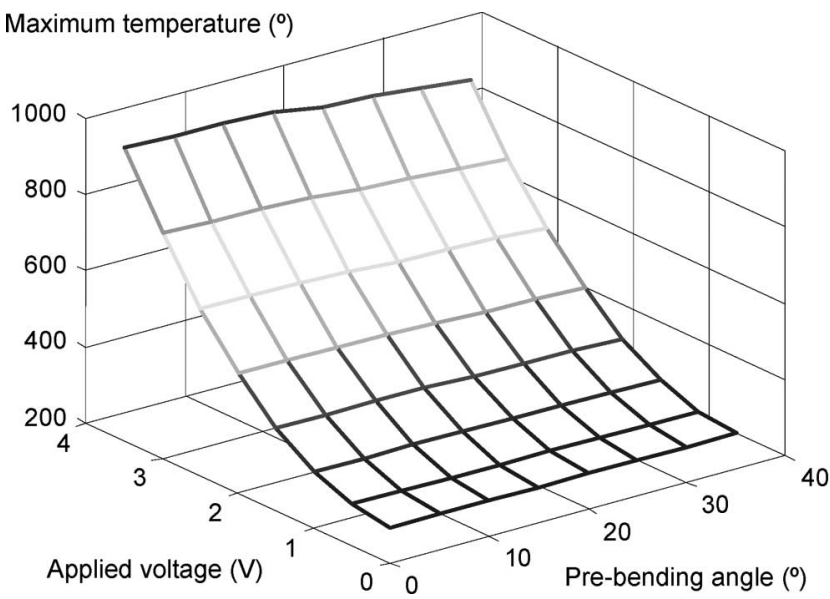

Fig. 9. Actuator maximum temperature as a function of the prebending angle $\varphi$ and the applied voltage.

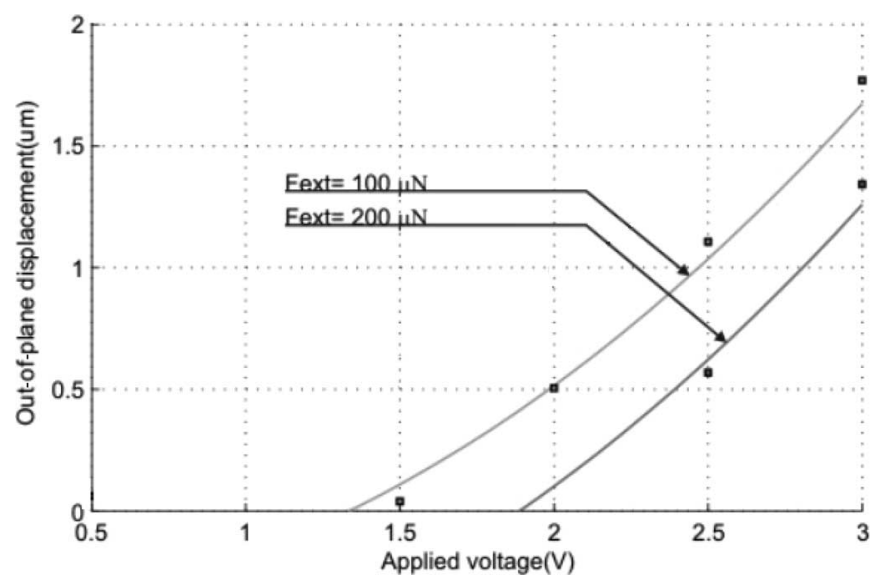

Fig. 10. Out-of-plane deflection of the VBB actuator with $\varphi=35^{\circ}$ for two different external forces applied to the tip of the actuator. Comparison between the analytical model (continuous lines) and FEA simulations done with CoventorWare (discrete points).

where $k_{\mathrm{VBB}}$ is the stiffness of VBB actuator, and $F_{\text {ext }}$ is the applied external force. In the case of the variable capacitor of the next section, $F_{\text {ext }}$, acting against the actuators, is due to the serpentine suspensions. In [24], Fedder proved that in case of a serpentine suspension, the force applied was linearly dependent on the deflection $\left(F_{\text {ext }}=-k_{s} \delta\right)$, where $k_{s}$ is the stiffness of the serpentine suspension. In Fig. 10, two different external loads of 100 and $200 \mu \mathrm{N}$ have been applied to the tip of a VBB actuator, with $\varphi=35^{\circ}$. As the external load is increased, the working range of the actuator is decreased, and more voltage is needed to achieve the same out-of-plane deflection.

As a first conclusion, a significant increase in displacement can be obtained by increasing the prebending angle $\varphi$. Fig. 8 shows that the actuator out-of-plane displacement for $\varphi=35^{\circ}$ is almost twice the displacement for $\varphi=0^{\circ}$. For instance, a simulated actuator displacement of $0.75 \mu \mathrm{m}$ can be obtained either with a prebending angle $\varphi=0^{\circ}$ and actuation voltage $V=2.32 \mathrm{~V}$ or with a prebending angle $\varphi=35^{\circ}$ and actuation voltage $V=1.66 \mathrm{~V}$, with a corresponding difference in the actuation current and temperature, which is an important feature in terms of thermal management, specifically in packaged devices.

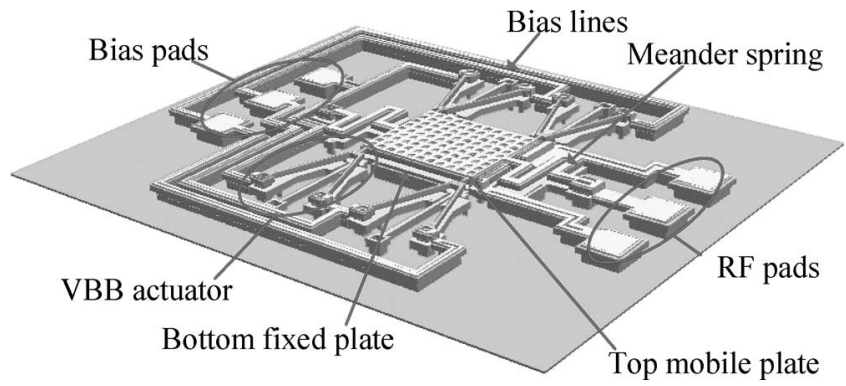

Fig. 11. 3-D view of a capacitor using four VBB as driving elements.

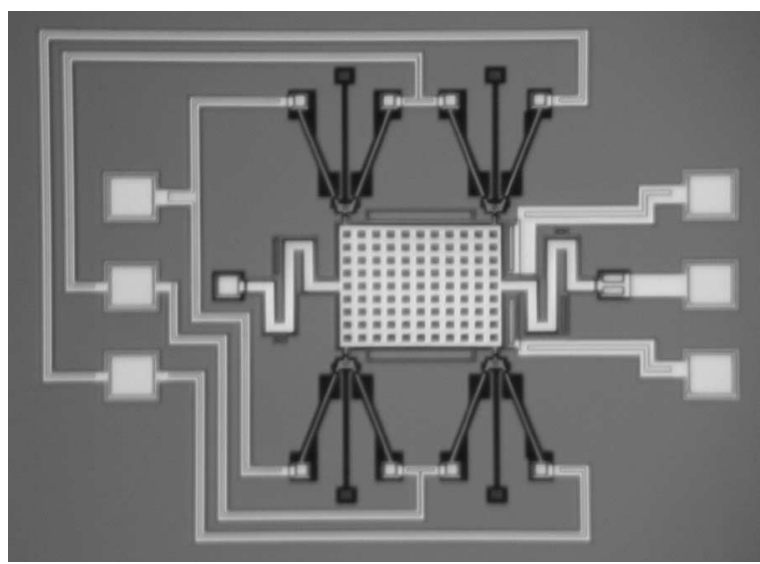

Fig. 12. Photograph of a manufactured capacitor using four VBB actuators with $\varphi=20^{\circ}$. Plate area: $260 \times 210 \mu \mathrm{m}^{2}$. Initial gap: $0.75 \mu \mathrm{m}$.

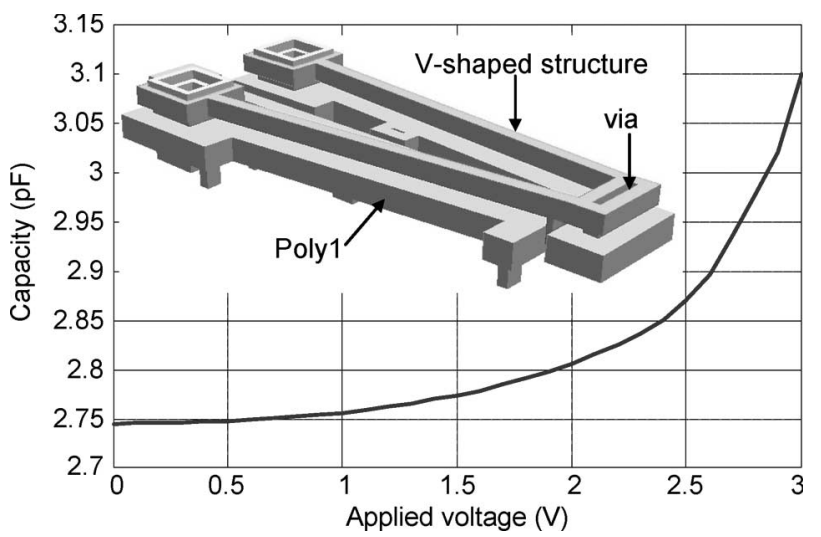

Fig. 13. Measured tuning range of the capacitor shown in Fig. 12.

In the above simulations, it can be observed that the mechanical models have the same temperature distributions, independent of the angle $\varphi$, since the other dimensions are exactly the same. However, it can be observed in Fig. 9 that the maximum temperature shows a slight tendency to decrease with an increasing $\varphi$. A feasible hypothesis for that is, since nonsquared meshing is not available in CoventorWare, the actuator 3-D model consists of an approximation built up with rectangles. This means that the actuator section is not constant, and therefore, small hot spots arise, which fix the maximum temperature. However, two additional observations are relevant. First, it has been assessed that the temperature distribution is identical in all the simulated devices, except in those small hot spots. Second, the decrease in the maximum temperature happens for large prebending angles $\varphi$, where simulations 


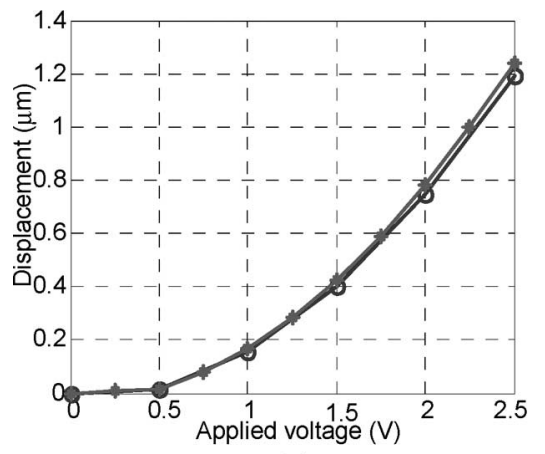

(a)

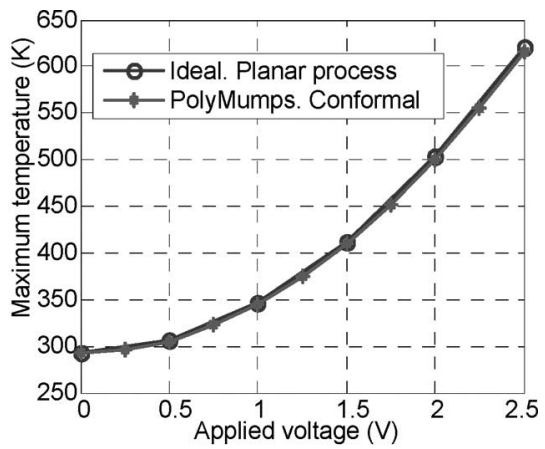

(b)

Fig. 14. Simulated comparison in terms of (a) displacement and (b) maximum temperature between the proposed ideal VBB shown in Fig. 1(a) (planar) and its adaptation to be manufactured with the PolyMumps conformal process (shown in Fig. 13).

demonstrate displacements much larger than those obtained with small $\varphi$ 's.

\section{RF CAPACITOR DRIVEN BY THE VBB ACTUATOR AND ACTUATOR VALIDATION}

This section explains the integration of the VBB actuator into a MEMS variable capacitor. As the goal now is to obtain an absolutely parallel-to-substrate motion, four VBB actuators are placed to control the squared top mobile membrane, one at each corner, as shown in the 3-D view of a designed capacitor shown in Fig. 11.

The designed capacitors have two different access pads: one for RF purposes (capacity variation testing) and another for actuator biasing. The holes in the top plate are for the proper release of the structures during sacrificial layer etching. A meander spring has been added in order to access the mobile membrane to measure the capacity variation; a second identical but reversed spring has been added at the other side of the mobile plate in order to have an absolutely balanced movement. Fig. 12 shows a photograph of a capacitor that was manufactured with PolyMumps [22]. Concerning the actuator design, it must be adapted to the PolyMumps conformal fabrication process and to its design rules. Therefore, some changes to the actuator shape are needed, resulting in the one shown in Fig. 13 (see insert). The first change is the use of a Poly1 element under the $\mathrm{V}$-shaped structure in order to ensure planarity. Second, a large via to connect the $\mathrm{V}$-shaped structure and the bottom cantilever is necessary in order to fulfill design rules. However, these two modifications do not imply significant differences in the actuator performance, as can be deduced from the comparison between an ideal VBB actuator [see Fig. 1(a)] and the one implemented with PolyMumps (see Fig. 13) shown in Fig. 14.

The correct performance of the proposed VBB actuator is deduced from the measured tuning range, shown in Fig. 13, of the capacitor in Fig. 12.

A comparison between three capacitors with the same dimensions but using three different $\operatorname{VBB}$ actuators $\left(\varphi=5^{\circ}, 20^{\circ}\right.$, and $35^{\circ}$ ) is given in Fig. 15, where their tuning ranges are shown. In order to perform a proper comparison, the parasitic capacity has been removed from the measured capacity and normalized to the estimated nominal capacity $C_{n}$ (case of no actuation, assuming $10 \%$ fringing capacity). The normalized

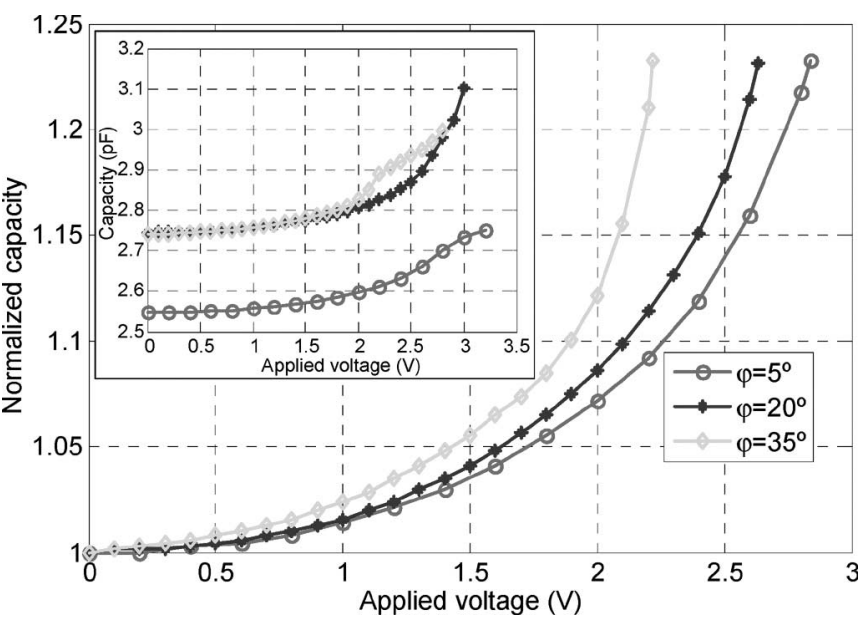

Fig. 15. Measured capacity variation (small plot) and comparison of normalized capacities for three parallel-plate capacitors driven by different VBB actuators with $\varphi=5^{\circ}, 20^{\circ}$, and $35^{\circ}$.

measured capacity is shown as a function of the actuation voltage, demonstrating a variation of $1.23: 1$ with respect to $C_{n}$. A quite illustrative result is obtained, demonstrating the VBB actuator usefulness and the advantages of increasing the angle $\varphi$. Note that the difference in the minimum capacity between the capacitor with $5^{\circ}$ and the other two is caused by a different parasitic capacity (the accesses are slightly different); nominal capacities and initial gaps are identical.

In Fig. 16, the improvement shown in Fig. 15 is quantified in terms of current and power consumptions. For instance, it can be assessed that a capacity variation of $1.23: 1$ with respect to $C_{n}$ can be obtained by using the three VBB actuators $(\varphi=$ $5^{\circ}, 20^{\circ}$, and $35^{\circ}$ ), with the actuation parameters summarized in Table III. Note that the power and current consumptions are the total values of the four driving actuators.

\section{CONCLUSion}

A new vertical electrothermal actuator has been proposed, which can be seen as a hybrid between the in-plane bucklebeam actuator and the vertical hot-cold actuator. It has been here referred to as VBB. Its performance has been analyzed, and its actuation principle has been shown, along with a study of its main parameters. An analytical model has been proposed and validated using FEA. It has been successfully integrated 


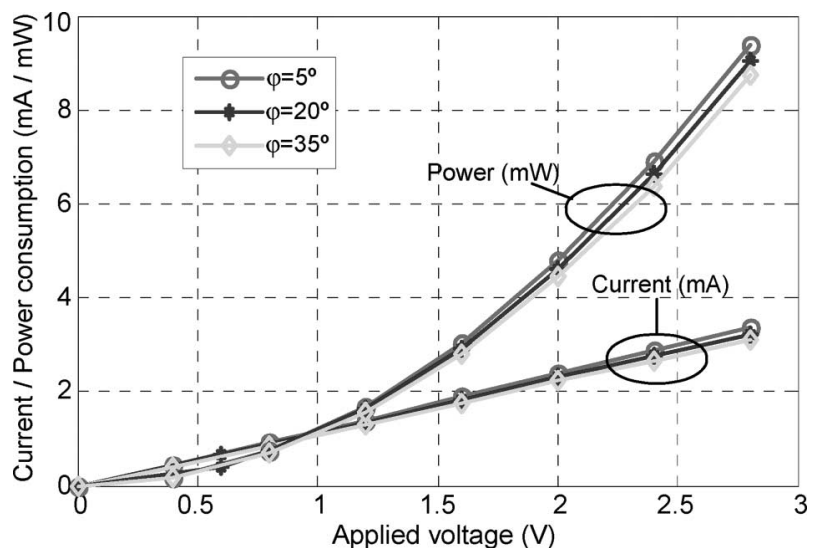

Fig. 16. Measured current and power consumptions for three parallel-plate capacitors driven by different VBB actuators with $\varphi=5^{\circ}, 20^{\circ}$, and $35^{\circ}$.

TABLE III

MEASUREd PARAMETERS

\begin{tabular}{lccc}
\hline \hline \multirow{2}{*}{ Parameter } & \multicolumn{3}{c}{$\varphi$} \\
\cline { 2 - 4 } & $5^{\circ}$ & $20^{\circ}$ & $35^{\circ}$ \\
\hline Tuning range & $1.23: 1$ & $1.23: 1$ & $1.23: 1$ \\
Actuation voltage (V) & 2.83 & 2.64 & 2.22 \\
Current consumption (mA) & 3.39 & 3.05 & 2.45 \\
Power consumption $(\mathrm{mW})$ & 9.59 & 8.09 & 5.5 \\
\hline \hline
\end{tabular}

into variable parallel-plate capacitors, demonstrating significant displacements, which increase as the initial prebending angle increases. This actuator has demonstrated potential dc power savings with respect to other vertical actuators presented in the literature so far.

\section{APPENDIX}

\section{COEFFicients of the Vertical Free Displacement} AND ROTATION ANGLE EQUATIONS

$$
\begin{aligned}
N_{1}= & -\frac{L_{3}^{2} h \cdot A_{3} L_{1}^{2}\left(L_{1}^{2} I_{3}+2 L_{3}^{2} I_{1}\right)}{2} \\
N_{2}= & \left(\left(-6 L_{1}^{2} L_{3}^{2}+8 L_{1} L_{3}^{3}\right) I_{1}+L_{1}^{4} I_{3}\right) r_{v} I_{3} A_{3} E \\
& +\left(I_{3} A_{3} h^{2}+I_{3}^{2}\right) L_{3} L_{1}^{4}-6 L_{1}^{2} L_{3}^{3} I_{1} I_{3} \\
& +\left(8 I_{3}+2 A_{3} h^{2}\right) L_{3}^{4} I_{1} L_{1} \\
N_{3}= & 2 I_{1} L_{3}\left(2 L_{3}^{3} I_{1}-3 L_{1}^{2} I_{3} L_{3}+4 L_{1}^{3} I_{3}\right) \\
& \times\left(L_{3}+A_{3} E r_{v}\right) \\
P_{1}= & -L_{3} h A_{3} L_{1}\left(L_{1}^{3} I_{3}+2 L_{3}^{3} I_{1}\right) \\
P_{2}= & A_{3}\left[\left(L_{1}^{4} I_{3}^{2}+8 L_{1} L_{3}^{3} I_{1} I_{3}-6 L_{1}^{2} L_{3}^{2} I_{1} I_{3}\right) r_{v} E\right. \\
& \left.+L_{1}^{4} I_{3} h^{2} L_{3}+2 L_{1} L_{3}^{4} h^{2} I_{1}\right] \\
& -6 L_{1}^{2} L_{3}^{3} I_{1} I_{3}+I_{3}^{2} L_{3} L_{1}^{4}+8 L_{3}^{4} I_{1} I_{3} L_{1} \\
P_{3}= & I_{1}\left[r_{v} E A_{3}\left(8 L_{1}^{3} I_{3} L_{3}+4 L_{3}^{4} I_{1}-6 L_{1}^{2} L_{3}^{2} I_{3}\right)\right. \\
& \left.+4 L_{3}^{5} I_{1}-6 L_{1}^{2} L_{3}^{3} I_{3}+8 L_{3}^{2} I_{3} L_{1}^{3}\right] .
\end{aligned}
$$

\section{ACKNOWLEDGMENT}

The authors would like to thank Dr. A. Viedma for his valuable advice in structural analysis.

\section{REFERENCES}

[1] A. Jain, H. Qu, S. Todd, G. K. Fedder, and H. Xie, "Electrothermal SCS micromirror with large-vertical-displacement actuation," in Proc. Solid-State Sensor, Actuator and Microsyst. Workshop, Jun. 2004, pp. 228-231.

[2] Y. Wang, Z. Li, D. T. McCormick, and N. C. Tien, "A low-voltage lateral MEMS switch with high RF performance," J. Microelectromech. Syst., vol. 13, no. 6, pp. 902-911, Dec. 2004.

[3] A. Jain and H. Xie, "An electrothermal microlens scanner with lowvoltage large-vertical-displacement actuation," IEEE Photon. Technol. Lett., vol. 17, no. 9, pp. 1971-1973, Sep. 2005.

[4] A. Atre, "Analysis of out-of-plane thermal microactuators," J. Micromech. Microeng., vol. 16, no. 2, pp. 205-213, Feb. 2006.

[5] J.-S. Park, L. L. Chu, A. D. Oliver, and Y. B. Gianchandani, "Bentbeam electrothermal actuators-Part II: Linear and rotary microengines," J. Microelectromech. Syt., vol. 10, no. 2, pp. 255-262, Jun. 2001.

[6] J. Qiu, J. H. Lang, A. H. Slocum, and A. C. Weber, "A bulkmicromachined bistable relay with U-shaped thermal actuators," J. Microelectromech. Syst., vol. 14, no. 5, pp. 1099-1109, Oct. 2005.

[7] Z. Feng, W. Zhang, B. Su, K. F. Harsh, K. C. Gupta, V. Bright, and Y. C. Lee, "Design and modeling of RF MEMS tunable capacitors using electro-thermal actuators," in Proc. IEEE MTT-S Int. Microw. Symp., Jun. 1999, vol. 4, pp. 1507-1510.

[8] H. D. Wu, K. F. Harsh, R. S. Irwin, W. Zhang, A. R. Mickelson, Y. C. Lee, and J. B. Dobsa, "MEMS designed for tunable capacitors," in Proc. IEEE MTT-S Int. Microw. Symp., Jun. 1998, vol. 1, pp. 127-129.

[9] W. Benecke and W. Riethmüller, "Applications of silicon-microactuators based on bimorph structures," in Proc. IEEE MEMS Workshop, Feb. 1989, pp. 116-120.

[10] S. Zhou, X.-Q. Sun, and W. N. Carr, "A micro variable inductor chip using MEMS relays," in Proc. IEEE Int. Conf. Solid-State Sens. and Actuators, Transducers, Jun. 1997, pp. 1137-1140.

[11] J. H. Comtois and V. M. Bright, "Applications for surface-micromachined polysilicon thermal actuators and arrays," Sens. Actuators A, Phys., vol. 58, no. 1, pp. 19-25, Jan. 1997.

[12] Z. Feng, H. Zhang, W. Zhang, B. Su, K. C. Gupta, V. M. Bright, and Y. C. Lee, "MEMS-based variable capacitor for millimeter-wave applications," in Proc. Solid-State Sensor and Actuator Workshop, Jun. 2000, pp. 255-258.

[13] W.-C. Chen, C.-C. Chu, J. Hsieh, and W. Fang, "A reliable single-layer out-of-plane micromachined thermal actuator," Sens. Actuators A. Phys., vol. 103, no. 1, pp. 48-58, Jan. 2003.

[14] D. Yan, A. Khajepour, and R. Mansour, "Design and modeling of a MEMS bidirectional vertical thermal actuator," J. Micromech. Microeng., vol. 14, no. 7, pp. 841-850, Jul. 2004.

[15] L. Que, J.-S. Park, and Y. B. Gianchandani, "Bent-beam electrothermal actuators-Part I: Single beam and cascaded devices," J. Microelectromech. Syst., vol. 10, no. 2, pp. 247-254, Jun. 2001.

[16] D. Girbau, A. Lázaro, and L. Pradell, "RF MEMS switches based on the buckle-beam thermal actuator," in Proc. 33rd Eur. Microw. Conf., Oct. 2003, vol. II, pp. 651-654.

[17] A. Dec and K. Suyama, "Micromachined electro-mechanically tunable capacitors and their applications to RF IC's," IEEE. Trans. Microw. Theory Tech., vol. 46, no. 12, pp. 2587-2596, Dec. 1998.

[18] D. Girbau, A. Lázaro, and L. Pradell, "Extended tuning range RF MEMS variable capacitors using electrostatic and electrothermal actuators," in Proc. SPIE-Micromachining and Microfabrication, Jan. 2004, vol. 5344, pp. 59-70.

[19] J. Zou, C. Liu, and J. Schutt-Ainé, "Development of a wide-tuning-range two-parallel-plate tunable capacitor for integrated wireless communication systems," Int. J. RF Microw. Comput.-Aided Eng., vol. 11, no. 5, pp. 322-329, Sep. 2001.

[20] D. Girbau, N. Otegi, L. Pradell, and A. Lázaro, "A MEMS capacitor with improved RF power handling capability," in Proc. 35th Eur. Microw. Conf., Oct. 2005, pp. 501-504.

[21] D. Girbau, N. Otegi, L. Pradell, and A. Lázaro, "Study of intermodulation in RF MEMS capacitors," IEEE Trans. Microw. Theory Tech., vol. 54, no. 3, pp. 1120-1130, Feb. 2006.

[22] D. Koester, A. Cowen, R. Mahadevan, M. Stonefield, and B. Hardy, PolyMUMPS Design Handbook. Revision 10.0. MEMSCAP, 2003. [Online]. Available: http://www.memscap.com/memsrus/svcsrules.html

[23] E. T. Enikov, S. S. Kedar, and K. V. Lazarov, "Analytical model for analysis and design of V-shaped thermal microactuators," J. Microelectromech. Syst., vol. 14, no. 4, pp. 788-798, Aug. 2005.

[24] G. K. Fedder, "Simulation of microelectromechanical systems," Ph.D. dissertation, Dept. Elect. Eng. Comput. Sci., Univ. California at Berkeley, Berkeley, CA, 1994. 


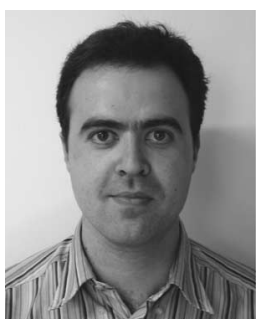

David Girbau received the Technical Telecommunication Engineering degree, the Electronics Engineering degree, and the Ph.D. degree in telecommunications from the Universitat Politécnica de Catalunya (UPC), Barcelona, Spain, in 1998, 2002, and 2006, respectively.

Since February 2001, he has been a Research Assistant in the Department of Signal Theory and Communications, UPC. Since September 2005, he has been a part-time Assistant Professor at the Universitat Autònoma de Barcelona. His primary research interests include RF and microwave devices and systems, with emphasis on RF-MEMS.

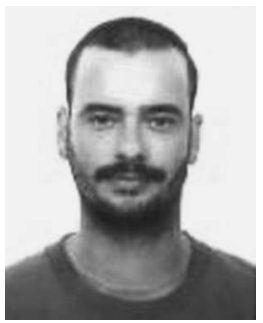

Marco Antonio Llamas was born in Spain. He received the Technical Telecommunication Engineering and Electronics Engineering degrees from the Universitat Politécnica de Catalunya, Barcelona, Spain, in 2001 and 2005, respectively. He is currently working toward the Ph.D. degree in the Department of Signal Theory and Communications, Universitat Politécnica de Catalunya.

His research is currently focusing on MEMS with emphasis on RF and microwave applications.

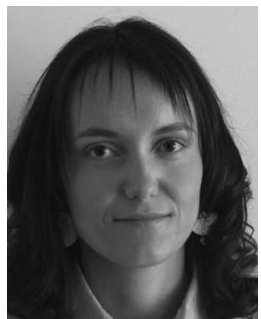

Jasmina Casals-Terré received the M.S. degree in mechanical and aerospace mechanical engineering from the University of California, Irvine, in 2002. She is currently working toward the Ph.D. degree in design, fabrication, and testing of bistable microswitches in the Department of Mechanical Engineering, Universitat Politécnica de Catalunya, Barcelona, Spain.

Her research interests are in bistable MEMS, such as switches or microvalves.

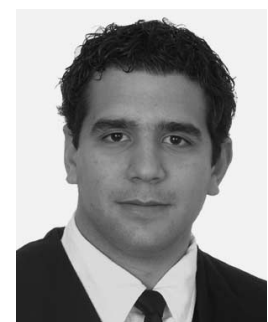

Xavier Simó-Selvas received the B.S. degree in mechanical engineering from the Universitat Politécnica de Catalunya, Barcelona, Spain, in 2004. He is currently working toward the M.S. degree in mechanical engineering in the Department of Mechanical Engineering, Universitat Politécnica de Catalunya.

During his M.S. degree program, he has worked on the VBB thermal actuator analysis, which is a project funded by a Spanish government fellowship.

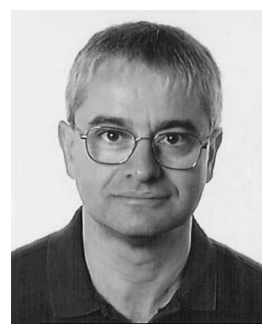

Lluís Pradell (M'87) was born in Barcelona, Spain, in 1956. He received the Technical Telecommunication Engineering degree from the Universitat Politécnica de Catalunya (UPC), Barcelona, in 1981 and the $\mathrm{Ph} . \mathrm{D}$. degree in telecommunication engineering from UPC in 1989.

From 1981 to 1985 , he was with Mier-Allende, Barcelona, as an RF and Microwave System Design Engineer. Since 1985, he has been with the Department of Signal Theory and Communications, UPC, where he became an Associate Professor in 1990. He has been teaching courses on microwave circuits and antennas, and performing research on models for microwave active devices (MESFET, HEMT, HBT), multimodal models for guiding structures and transitions (microstrip, finline, slotline, CPW), on-wafer measurement techniques (network-analyzer calibration, noise parameters), development of microwave and millimeter-wave systems (low-noise amplifiers and point-to-multipoint broadband communication systems), and RF and microwave microelectromechanical systems, in the frequency range $1-75 \mathrm{GHz}$.

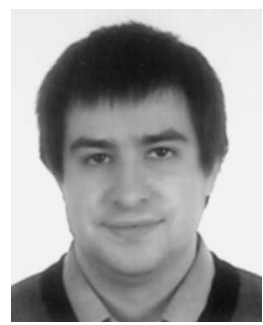

Antonio Lázaro was born in Lleida, Spain, in 1971. He received the M.S. and Ph.D. degrees in telecommunication engineering from the Universitat Politécnica de Catalunya (UPC), Barcelona, Spain, in 1994 and 1998, respectively.

$\mathrm{He}$ then joined the faculty at UPC, where he taught a course on microwave circuits and antennas. $\mathrm{He}$ is currently with the Department of Electronics, Electrical and Automatics Engineering, University Rovira i Virgili, Tarragona, Spain. His research interests are microwave device modeling, on-wafer noise measurements, monolithic microwave integrated circuits, low phase noise oscillators, MEMS, and microwave systems. 